ORIGINAL ARTICLE

\title{
Neurodevelopmental outcome of hypoglycaemia in healthy, large for gestational age, term newborns
}

\author{
P L P Brand, N L D Molenaar, C Kaaijk, W S Wierenga
}

Arch Dis Child 2005;90:78-81. doi: 10.1136/adc.2003.039412

\begin{abstract}
See end of article for authors' affiliations

Correspondence to

Dr P L P Brand, Department

of Paediatrics, Isala klinieken, PO Box 10500, 8000 GM Zwolle, Netherlands; p.l.p.brand@ isala.nl
\end{abstract}

Accepted 6 April 2004

\begin{abstract}
Aims: To evaluate the effects of transient hypoglycaemia on the first day of life in 75 healthy term large for gestational age (LGA) infants, born to non-diabetic mothers, on their neurodevelopmental outcome at the age of 4 years.

Methods: Screening for hypoglycaemia was performed 1, 3, and 5 hours after birth, and continued if blood glucose levels were low. Treatment with intravenous glucose for hypoglycaemia was started if hypoglycaemia was severe or symptomatic. Patients' development and behaviour was examined at the age of 4 years by the Denver Developmental Scale, a non-verbal intelligence test, and the Child Behaviour Check List.

Results: There were no significant differences between children with neonatal normoglycaemia $(n=15)$ and hypoglycaemia (plasma glucose $<2.2 \mathrm{mmol} / \mathrm{l} 1$ hour after birth, or $<2.5 \mathrm{mmol} / \mathrm{l}$ subsequently; $n=60$ ) in Denver developmental scale scores and child behaviour checklist scores. Although total IQ did not differ between hypoglycaemic and normoglycaemic children, one subscale (reasoning) did (mean difference $9.3,95 \% \mathrm{Cl} 1.3$ to 17.2). The correlation between reasoning IQ and neonatal blood glucose levels was weak and not statistically significant. When other definitions for hypoglycaemia were applied, the difference in reasoning IQ was not found. There were no differences in any of the test scores between hypoglycaemic children who had and who had not been treated with intravenous glucose.

Conclusion: Transient mild hypoglycaemia in healthy, term LGA newborns does not appear to be harmful to psychomotor development at the age of 4 years.
\end{abstract}

ypoglycaemia is a common finding in newborns, in particular on the first day of life. ${ }^{1}$ The risk of hypoglycaemia is increased in premature and small for gestational age (SGA) newborns, in infants of mothers with insulin dependent or gestational diabetes, and in infants with hyperinsulinaemia. ${ }^{1-4}$ Follow up studies of hypoglycaemic infants from these risk groups have shown important neurodevelopmental deficits, ${ }^{5-8}$ particularly in symptomatic infants. ${ }^{68}$

Hypoglycaemia may also occur in up to $14 \%$ of healthy term newborns, ${ }^{9-12}$ and in $16 \%$ of large for gestational age (LGA) term newborns ${ }^{13}$ from non-diabetic mothers. ${ }^{14}$

Management of hypoglycaemia in term infants is controversial. While it is assumed by many that hypoglycaemia in healthy term newborns is a harmless finding not associated with impaired psychomotor development, ${ }^{19}$ both radiological and clinical neurological sequelae have been described in term infants with severe or symptomatic hypoglycaemia of unknown cause. ${ }^{415} 16$ Screening for hypoglycaemia has been proposed as a routine procedure for healthy term LGA newborns, based on the assumption that being LGA in newborns may be associated with organic hyperinsulinism or hitherto undetected maternal diabetes. ${ }^{14}$

The lack of studies comparing neurodevelopmental outcome between term newborns with and without hypoglycaemia hinders meaningful interpretation of blood glucose values in these infants. ${ }^{17}$ We conducted this study to examine the effects on neurodevelopmental outcome of hypoglycaemia and its treatment during the first day of life in healthy, term LGA newborns.

\section{METHODS}

\section{Patients}

From the birth data of all infants, born between 1 January 1997 and 1 January1998 in our hospital (a general district hospital with an average of 1100 births per year), LGA term (born after 37 weeks of gestation or more) infants were identified, and the hospital records of these infants and their mothers were reviewed retrospectively. LGA was defined as birth weight exceeding the 90th centile for gestational age (adjusted for parity of the mother and gender of the infant), according to Dutch standards (which were published in 1969 and revalidated in 1990). ${ }^{18}$ Infants were excluded if maternal or infant records showed evidence of maternal insulin dependent or gestational diabetes or severe perinatal asphyxia (Apgar score $<3$ after 1 minute or $<7$ after 5 minutes). In 1997, no term LGA infants born in our hospital suffered from severe and prolonged symptomatic hypoglycaemia, associated with hyperinsulinism, nor from recurrent hypoglycaemic episodes.

\section{Data drawn from hospital records}

Gestational age, parity, method of birth, sex, birth weight, and neonatal clinical course were recorded for all infants. In 1997, the practice guideline in our hospital was to obtain blood glucose levels 1,3 , and 5 hours after birth in infants considered to be at risk for neonatal hypoglycaemia (including term LGA newborns), and to follow up glucose levels if the initial screening revealed hypoglycaemia (defined as $<2.2 \mathrm{mmol} / \mathrm{l}(40 \mathrm{mg} / \mathrm{dl})$ in the first hour after birth and $<2.5 \mathrm{mmol} / \mathrm{l}$ (46 mg/dl) subsequently). Results of blood glucose levels were obtained from hospital records and cross checked with computer records of the hospital laboratory. Treatment of hypoglycaemia was not standardised. The usual policy was to recommend additional feeding with mild (>1.5-2 mmol/l) and asymptomatic hypoglycaemia, and to start intravenous glucose with severe $(<1.5-2 \mathrm{mmol} / \mathrm{l})$ or symptomatic hypoglycaemia.

\section{Blood glucose concentrations}

Whole blood samples for glucose concentrations were obtained by heel prick after warming the infant's foot. Plasma glucose concentrations were determined within 30 minutes of obtaining the blood sample by a glucose oxidase method (2300 STAT+YSI analyser, Yellow Springs Instruments, Yellow Springs, $\mathrm{OH}$ ). 


\section{Follow up and neurodevelopmental testing}

In the autumn of 2000, the current addresses of the healthy term LGA infants born in our hospital in 1997 were traced through municipal address registries, and the patients and their parents were invited to return to the hospital for neurodevelopmental testing when the child was 4 years of age. Parents who did not respond to repeated written invitations were contacted by telephone and asked to participate. All participating children were examined by the same, experienced psychological test assistant (CK) who was blinded to the neonatal history of the patients. The children were tested with the Dutch version ${ }^{20}$ of the Denver Developmental Scale (DDS) ${ }^{21}$ and the Snijders-Oomen non-verbal intelligence test $(\mathrm{SON}),{ }^{22}$ and the parents completed the Dutch version of the Child Behaviour Check List (CBCL). ${ }^{23}$

The DDS is a 105 item development screening instrument with four subscales of behaviour: social, adaptive, language, and motor behaviour. These scales are scored as definitively normal, possibly abnormal, and definitively abnormal. The SON is a validated general non-verbal intelligence test designed for Dutch children between $2 \frac{1}{2}$ and 7 years of age. Next to a total intelligence quotient (IQ), IQs for the subscales reasoning and spatial orientation can be derived. The CBCL is designed as a screening instrument for behavioural disorders, and rated as a total score and eight subscales (withdrawal, physical complaints, anxiety/depression, social, thinking, attention problems, and delinquent and aggressive behaviour).

\section{Analysis}

Children were categorised in three groups. Children in whom blood glucose concentrations remained normal (that is, above the hypoglycaemic range defined above) were classified as normoglycaemic. Infants with hypoglycaemia during the first day of life were classified as treated when they had received therapy with intravenous glucose, and as untreated when they had not. Because there is considerable uncertainty as to the definition of hypoglycaemia in newborn infants, ${ }^{12} 924$ other definitions of hypoglycaemia were also applied to this classification.

Differences in outcome parameters between study groups were analysed by $\chi^{2}$ testing for categorised variables, and by Student's $t$ tests and analysis of variance for continuous variables. Two sided $p$ values $<0.05$ were considered statistically significant. Statistics were performed using SPSS for Windows version 8.0-1 and confidence interval analysis (CIA).

\section{Ethical considerations}

The study was approved by the hospital's ethical review board. All parents gave written informed consent.

\section{RESULTS}

\section{Patients}

Figure 1 presents an account of the entire population of 117 eligible LGA infants. The practice guideline of obtaining and monitoring blood glucose levels after birth in LGA infants was followed in $87 \%$ of infants. Some addresses could not be traced because parents divorced or moved abroad; a number of other infants had to be excluded because parents refused consent. Of the 117 healthy term LGA newborns born in our hospital in 1997, 75 were tested completely and reliably at the age of 4 years (fig 1). These 75 tested infants were comparable to the root population of 117 infants in terms of gestational age, birth weight, gender, parity, blood glucose levels, frequency of hypoglycaemia, frequency of intravenous glucose therapy and socioeconomic status (data not shown; all $\mathrm{p}>0.3$ ). Table 1 gives characteristics of the study population of 75 fully tested children. Only three children (4\%) were exclusively breast fed during the first three days of life; the

\section{LGA term newborns}

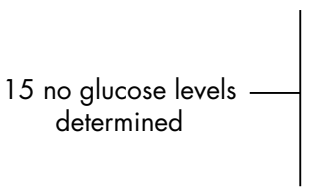

102 with glucose levels checked post partum

1 child not tested (severe mental deficiency, unrelated cause)

Figure 1 Composition of study population from original population of 117 healthy term LGA newborns. The study population of 75 tested children was representative of the root population with respect to demographic, socioeconomic, and clinical characteristics (including blood glucose levels).

remaining children were either formula fed $(n=19,25 \%)$ or received a combination of breast and formula feeding.

\section{Glucose levels and glucose therapy}

Distributions of plasma glucose levels are given in table 1, and the number and percentage of hypoglycaemic children (using different definitions of hypoglycaemia) in table 2 . Hypoglycaemia was very common among the 75 LGA children. The lowest blood glucose levels observed during the first five hours of life ranged from 0.6 to $3.7 \mathrm{mmol} / \mathrm{l}$ (mean $1.9 \mathrm{mmol} / \mathrm{l})$. Twenty seven children (36\%) were treated with intravenous glucose infusion for a mean duration of 2.0 days (range 0.5-4 days). Plasma glucose levels on the first day of life were lower in the 29 hypoglycaemic children who were treated with intravenous glucose (mean 1.4, SD $0.6 \mathrm{mmol} / \mathrm{l}$ ) than in the 46 who were not $(2.1, \mathrm{SD} 0.4 ; 95 \% \mathrm{CI}$ for difference 0.4 to $0.9 \mathrm{mmol} / \mathrm{l})$.

\section{Results of psychological tests at the age of 4 years}

Total and most subscale DDS scores were definitively normal in $>90 \%$ of children. Twenty two children (29\%) had possibly abnormal motor behaviour, two were definitively abnormal

Table 1 Demographic and clinical characteristics of 75 healthy term LGA newborns

\begin{tabular}{ll}
\hline Characteristic & No. (\%) \\
\hline Male sex (\%) & $39(51 \%)$ \\
Firstborn (\%) & $42(55 \%)$ \\
Birth & $32(42 \%)$ \\
Spontaneous (\%) & $23(30 \%)$ \\
Vacuum extraction (\%) & $4(5 \%)$ \\
Forceps extraction (\%) & $17(22 \%)$ \\
Caesarean section & $4262(250)$ \\
Mean birth weight in grams (SD) & $27(36 \%)$ \\
$>$ P97.7 (\%) & $25(33 \%)$ \\
P95-97.7 (\%) & $23(31 \%)$ \\
P90-95 (\%) & \\
Mean whole blood glucose in mmol/l & \\
(SD, range) & $2.42(1.07,0.6-5.8)$ \\
1 h after birth & $2.57(0.62,1.0-4.0)$ \\
3 h after birth & $2.47(0.63,0.8-3.9)$ \\
5 h after birth & \\
\hline &
\end{tabular}


Table 2 Number and percentage of children with hypoglycaemia according to different definitions in 75 healthy term LGA newborns

\begin{tabular}{|c|c|c|c|}
\hline Reference & Definition of hypoglycaemia (in $\mathrm{mmol} / \mathrm{l}$ ) & $\begin{array}{l}\text { No. of children with } \\
\text { hypoglycaemia }\end{array}$ & $\begin{array}{l}\% \text { of children with } \\
\text { hypoglycaemia }\end{array}$ \\
\hline $\begin{array}{l}\text { Zwolle } \\
\text { Srinivasan et } a l^{12} \\
\text { Cornblath et } a l^{2}\end{array}$ & $\begin{array}{l}<2.21 \text { h after birth, }<2.5 \text { subsequently } \\
<2.2 \text { during the } 1 \text { st day of life, }<2.5 \text { subsequently } \\
<2.5 \text { at any time point } \\
<2.0 \text { at any time point } \\
<1.4 \text { at any time point }\end{array}$ & $\begin{array}{l}60 \\
34 \\
66 \\
42 \\
14\end{array}$ & $\begin{array}{l}80 \% \\
45 \% \\
88 \% \\
56 \% \\
19 \%\end{array}$ \\
\hline
\end{tabular}

$(3 \%)$. Both the total and the subscale scores of the CBCL were normal in $>90 \%$ of patients. The mean SON total IQ score was 109.2 (SD 15.7), with comparable results for both subscales.

\section{Comparison of psychological test scores between children with and without hypoglycaemia}

Using the Zwolle definition for hypoglycaemia (table 2), there were no significant differences between children with and without hypoglycaemia in DDS, CBCL, and total SON-IQ scores (table 3). There was, however, a statistically significant difference in the SON reasoning IQ between children with hypoglycaemia (mean (SD) 107.6 (15.0)) and those without hypoglycaemia (116.9 (16.1), 95\% CI for difference 1.3 to $17.2, \mathrm{p}=0.023)$, which remained unchanged after adjusting for perinatal complications (Apgar score $<7$ after 1 minute, hyperbilirubinaemia, use of antibiotics, or supplemental oxygen in the first 72 hours of life) in a multiple regression model. No significant correlation was found between the lowest plasma glucose level and the SON reasoning IQ score $(r=0.11, \mathrm{p}=0.33)$.

When using other definitions for hypoglycaemia (table 2), there no longer was a significant difference in SON reasoning score between hypoglycaemic and normoglycaemic infants (all $\mathrm{p}>0.2$ ). Using the Srinivasan or Cornblath definitions of hypoglycaemia as a blood glucose level $<2.0$ or $1.4 \mathrm{mmol} / \mathrm{l}$ (table 2), ${ }^{2}{ }^{12}$ no significant differences between hypoglycaemic and normoglycaemic infants were found in any of the psychological test scores at 4 years. When using the $<2.5 \mathrm{mmol} / \mathrm{l}$ criterion, children in the normoglycaemic group had higher scores on the CBCL total $(p=0.04)$ and externalising behaviour subscale $(\mathrm{p}=0.01)$ than the hypoglycaemic children, indicating a poorer result in the normoglycaemic children.

\section{Comparison of psychological test scores between hypoglycaemic children with and without intravenous glucose therapy}

Of the 60 children with hypoglycaemia, 27 (45\%) had been treated with intravenous glucose. There were no differences in psychological test scores between hypoglycaemic children who had and who had not received intravenous glucose therapy (all p >0.2).

\section{DISCUSSION}

This study is the first to examine the neurodevelopmental outcome at 4 years of age in a group of healthy, term LGA newborns with transient hypoglycaemia during the first day of life. In the vast majority of neurodevelopmental and behavioural tests at follow up, there were no significant differences between children with and without hypoglycaemia. The only significant difference observed was in one subscale of the SON intelligence test, namely the reasoning score, which was on average 9.2 points higher in the normoglycaemic group than in the hypoglycaemic group. Such a difference in a subscale of a test, without any difference in the test's total score should be interpreted very cautiously.
We argued that the difference in SON reasoning scores at 4 years between normoglycaemic and hypoglycaemic newborns was more likely to be real if it remained significant after adjustment for other possible neonatal causes of reduced IQ at the age of 4 (perinatal asphyxia and hypoxia, hyperbilirubinaemia, and perinatal infection), was robust to using different definitions of hypoglycaemia, and if the SON reasoning score showed a linear relation to the lowest glucose level found in individual patients. Although the former appeared to be the case, none of the two latter was. When using other definitions of hypoglycaemia (table 2), the significant difference in SON reasoning IQ scores between normoglycaemic and hypoglycaemic newborns disappeared, and the only other difference observed was one in CBCL scores (total score and one subscale), which was in the opposite direction as expected (poorer scores in the normoglycaemic group). In addition, no correlation was found between the lowest glucose level and the SON scores in individual subjects. This suggests that mild and transient hypoglycaemia in healthy term LGA newborns in the first few days of life is not associated with clinically relevant neurodevelopmental sequelae at the age of 4 years.

The limitations of our study design should be borne in mind when interpreting its results. Firstly, it was a retrospective chart review study which always carries the risk of incomplete or inaccurate data. Because the data on gestational age, birth weight, and neonatal glucose were recorded prospectively and in computerised records, it is unlikely, however, that this had a major influence on our results. Moreover, all 75 infants whom we examined at follow up had complete data on glucose levels at 1, 3, and 5 hours after birth. Data on symptoms of hypoglycaemia and motivation for withholding or starting intravenous glucose treatment are unavailable, however.

Secondly, our study sample was relatively small. Because there was a trend towards poorer outcome in hypoglycaemic LGA newborns (table 3), a larger study sample might have resulted in more significant differences between groups.

Thirdly, because only $64 \%$ of the original population of 117 LGA infants was followed up with neurodevelopmental testing at the age of 4 years, the possibility of selection bias should be considered. The examined cohort was representative of the original cohort in terms of demographic, socioeconomic, and birth characteristics. Even if those who did not consent to follow up were more likely to have a worse neurodevelopmental outcome, this would not have influenced the relation between neonatal hypoglycaemia and outcome, because the frequency of hypoglycaemia was comparable between the children who were and who were not followed up at 4 years of age.

A final limitation is that we used the DDS instead of the more comprehensive and sensitive Bayley Scales of Infant Development (BSID). This was done for practical reasons. The DDS takes approximately 15 minutes to administer, whereas the BSID takes approximately 40 minutes. We used an intelligence test as well, and the SON test takes 50-60 
Table 3 Results of psychological tests at the age of 4 years in 75 healthy term LGA newborns with or without hypoglycaemia

\begin{tabular}{l}
\hline Test or subscale \\
\hline DDS total score (\% definitively normal) \\
DDS social behaviour (\% definitively nor \\
DDS adaptive behaviour (\% definitively nor \\
DDS language behaviour (\% definitively \\
DDS motor behaviour (\% definitively nor \\
CBCL withdrawal (\% normal) \\
CBCL physical complaints (\% normal) \\
CBCL anxiety/depression (\% normal) \\
CBCL social problems (\% normal) \\
CBCL thinking problems (\% normal) \\
CBCL attention problems (\% normal) \\
CBCL delinquent behaviour (\% normal) \\
CBCL aggressive behaviour (\% normal) \\
CBCL internalising T score, mean (SD) \\
$C B C L$ externalising T score, mean (SD) \\
$C B C L$ total T score, mean (SD) \\
SON spatial orientation IQ, mean (SD) \\
SON reasoning IQ, mean (SD) \\
SON total IQ, mean (SD)
\end{tabular}

\begin{tabular}{lcl} 
Normoglycaemic children & Hypoglycaemic children & $\mathbf{9 5 \%}$ Cl for difference \\
$100 \%$ & $93 \%$ & -14 to $16 \%$ \\
$100 \%$ & $93 \%$ & -14 to $16 \%$ \\
$100 \%$ & $93 \%$ & -14 to $16 \%$ \\
$100 \%$ & $97 \%$ & -17 to $11 \%$ \\
$67 \%$ & $68 \%$ & -28 to $20 \%$ \\
$100 \%$ & $95 \%$ & -16 to $14 \%$ \\
$100 \%$ & $95 \%$ & -16 to $14 \%$ \\
$100 \%$ & $97 \%$ & -17 to $11 \%$ \\
$100 \%$ & $98 \%$ & -19 to $9 \%$ \\
$93 \%$ & $93 \%$ & -24 to $11 \%$ \\
$93 \%$ & $97 \%$ & -27 to $7 \%$ \\
$87 \%$ & $98 \%$ & -36 to $1 \%$ \\
$87 \%$ & $90 \%$ & -29 to $11 \%$ \\
$48.4(10.6)$ & $50.4(10.7)$ & -8.1 to 4.2 \\
$54.7(12.4)$ & $51.4(10.7)$ & -3.1 to 9.6 \\
$52.9(10.8)$ & $51.3(11.7)$ & -5.0 to 8.2 \\
$108.9(12.8)$ & $108.2(16.3)$ & -8.3 to 9.7 \\
$116.8(16.1)$ & $107.6(13.2)$ & 1.3 to 17.2 \\
$114.3(14.5)$ & $108.9(14.1)$ & -2.8 to 13.5 \\
\hline
\end{tabular}

Hypoglycaemia defined as a blood glucose level $<2.2 \mathrm{mmol} / \mathrm{I}$ during the first hour after birth, and $<2.5 \mathrm{mmol} / \mathrm{l}$ afterwards (Zwolle criterion, see table 2) Results are presented as percentages for proportions, and as mean (SD) for continuous variables.

minutes to administer. Because of the limited time span younger children can be tested in, it would have taken two separate sessions to administer both SON and BSID, whereas SON and DDS could be combined in a single session, which we preferred in order to increase the willingness of parents to return to our hospital for testing. Our results with normal DDS scores in almost all children (table 3) suggest that the DDS may not be sensitive enough as a follow up instrument for studies on neurodevelopmental sequelae of neonatal hypoglycaemia.

One of the reasons why blood glucose monitoring is commonly performed in LGA newborns is the assumption that being LGA may be a presenting symptom of hyperinsulinaemia. This, however, does not appear to be the case. None of the LGA newborns in our study group had persistent or recurrent hypoglycaemia or evidence of hyperinsulinaemia. Other studies have shown that only $20 \%$ of infants with hyperinsulinaemia are LGA at birth. ${ }^{25}$

Our study supports the recent advice to refrain from routine blood glucose measurements in healthy term infants who are not small for gestational age. ${ }^{9}$ Our study sample comprised infants born in hospital. In the Netherlands, many healthy infants from uncomplicated pregnancies are born at home, and a hospital based population of term infants contains an overrepresentation of infants at increased risk of perinatal complications and neurodevelopmental sequelae. The finding that even in this population, hypoglycaemia in healthy term LGA infants appears not to be associated with poor neurodevelopmental outcome at the age of 4 years is, therefore, particularly reassuring.

\section{Authors' affiliations}

P L P Brand, N L D Molenaar, Department of Paediatrics, Isala klinieken, PO Box 10500, 8000 GM Zwolle, Netherlands

C Kaaijk, W S Wierenga, Department of Medical Psychology, Isala klinieken, PO Box 10500, 8000 GM Zwolle, Netherlands

Supported by a grant from the Innovation of Care Project of the Isala klinieken, Zwolle, Netherlands

\section{REFERENCES}

1 World Health Organisation. Hypoglycaemia of the newborn. Review of the literature. WHO/CHD/97.1, available at http://www.who.int. 1997. Geneva, World Health Organisation.

2 Cornblath M, Hawdon JM, Williams AF, et al. Controversies regarding definition of neonatal hypoglycemia: suggested operational thresholds. Pediatrics 2000;105:1141-5.
3 Hawdon JM, Ward-Platt MP, Aynsley-Green A. Patterns of metabolic adaptation for preterm and term infants in the first neonatal week. Arch Dis Child 1992;67:357-65.

4 Dalgic N, Ergenekon E, Soysal S, et al. Transient neonatal hypoglycemialong-term effects on neurodevelopmental outcome. J Pediatr Endocrinol Metab 2002; 15:319-24.

5 Bosson Duvanel C, Fawer C-L, Cotting J, et al. Long-term effects of neonatal hypoglycemia on brain growth and psychomotor development in small-forgestational-age preterm infants. J Pediatr 1999;134:492-8.

6 Koivisto M, Blanco-Sequeiros M, Krause U. Neonatal symptomatic and asymptomatic hypoglycaemia: a follow-up study of 151 children. Dev Med Child Neurol 1972;14:603-14.

7 Lucas A, Morley R, Cole TJ. Adverse neurodevelopmental outcome of moderate neonatal hypoglycaemia. BMJ 1988;297:1304-8.

8 Pildes RS, Cornblath M, Warren I, et al. A prospective controlled study of neonatal hypoglycemia. Pediatrics 1974:54:5-14.

9 Nicholl R. What is the normal range of blood glucose concentrations in healthy term newborns? Arch Dis Child 2003;88:238-9.

10 Hoseth $E$, Joergensen A, Ebbesen F, et al. Blood glucose levels in a population of healthy, breast fed, term infants of appropriate size for gestational age. Arch Dis Child Fetal Neonatal Ed 2001;83:F117-19.

11 Heck $L$, Erenberg A. Serum glucose levels in term neonates during the first 48 hours of life. J Pediatr 1987; 110:119-22.

12 Srinivasan G, Pildes RS, Cattamanchi G, et al. Plasma glucose values in normal neonates: a new look. J Pediatr 1986;109:114-17.

13 Holtrop PC. The frequency of hypoglycemia in full-term large and small for gestational age newborns. Am J Perinatol 1993;10:150-4.

14 Schaefer-Graf UM, Rossi R, Buhrer C, et al. Rate and risk factors of hypoglycemia in large-for-gestational-age newborn infants of nondiabetic mothers. Am J Obstet Gynecol 2002;187:913-17.

15 Murakami Y, Yamashita Y, Matsuishi T, et al. Cranial MRI of neurologically impaired children suffering from neonatal hypoglycemia. Pediatr Radiol 1999;29:23-7.

16 Kinnala A, Rikalainen $\mathrm{H}$, Lapinleimu $\mathrm{H}$, et al. Cerebral magnetic resonance imaging and ultrasonography findings after neonatal hypoglycemia. Pediatrics 1999:103:724-9.

17 Brand PLP. What is the normal range of blood glucose concentrations in healthy term newborns? Arch Dis Child Fetal Neonatal Ed 2004;89:F373-6.

18 Kloosterman GJ. Intrauterine growth and intrauterine growth curves. Maandschr Kindergeneeskd 1969;37:209-25.

19 Voorhorst FJ, Puyenbroek Jl, Robertson EA, et al. Are earlier birth weights different from current ones? (in Dutch). Ned Tijdschr Geneeskd 1990;134:998-1002.

20 Cools ATM, Hermanns JMA. DOS Handleiding. Amsterdam: Swets \& Zeitlinger, 1979.

21 Frankenburg WK, Camp BW, Natta PAv. Validity of the Denver developmental screening test. Child Dev 1971;42:475-85.

22 Tellegen PJ, Winkel M, Wijnberg-Williams BJ, et al. Snijders-Oomen nietverbale intelligentieschaal, SON-R $2 \frac{1}{2}-7$. Handleiding. Lisse: Swets \& Zeitlinger, 1998.

23 Verhulst FC, Althaus M. Persistence and change in behavioral/emotional problems reported by parents of children aged 4-14: an epidemiological study. Acta Psychiatr Scand Suppl 1988;339:1-28.

24 Cornblath M, Ichord R. Hypoglycemia in the neonate. Semin Perinatol $2001 ; 24: 136-49$

25 Tyrrell VJ, Ambler GR, Yeow W-H, et al. Ten years' experience of persistent hyperinsulinaemic hypoglycaemia of infancy. J Paediatr Child Health 2001;37:483-8. 